\title{
Oralität und Literalität: Wie polnische Deutschlerner den Unterschied zwischen gesprochener und geschriebener Sprache auf Textebene wahrnehmen
}

\author{
Orality and literality: How Polish learners \\ of German perceive the distinction between \\ spoken and written language on text level
}

\begin{abstract}
The present paper deals with both medium and conception of orality and literality on text level, and aims at giving an outline of some important aspects underlying the discussion about the differences between written and spoken language and their impact on Polish learners of German.

KEYWORDS: spoken language (orality), written language (literality), medium, conception, foreign language writing.
\end{abstract}

Im Rahmen der nachstehenden Ausführungen wird die Absicht verfolgt, einerseits wesentliche Aspekte aus der Diskussion um die Unterschiede zwischen gesprochener und geschriebener Sprache herauszustellen und andererseits aufzuzeigen, inwieweit polnische Lerner des Deutschen als Fremdsprache im (schulischen) Unterricht auf die Besonderheiten der beiden hingewiesen werden und ob und inwieweit sie sich dieser bewusst sind.

Der Ausdruck Schriftlichkeit wird in verschiedenen älteren Lexika ähnlich wie bei Bußmann (1983) definiert, wo folgende Definition von Schrift angegeben wird: „auf konventionalisiertem System von graphischen Zeichen basierendes Mittel zur Aufzeichnung von mündlicher Sprache“. Die Auffassung, wonach die Schrift nur das eigentliche, die mündliche Sprache, in ein anderes 
Medium überträgt, dass Schrift „nicht Sprache, sondern nur ein Mittel, Sprache mit Hilfe sichtbarer Kennungen aufzuzeichnen, ist", die der amerikanische Linguist Bloomfield (1933) in seinem Buch Language zum Ausdruck gebracht hat, ist die opinio communis der letzten 2000 Jahre nicht nur in der Sprachwissenschaft (Günther 2010: 9). Allerdings hat man diese Perspektive in den 80er und 90er Jahren des vergangenen Jahrhunderts einer zunehmenden Kritik unterzogen und an der Auffassung, wonach die Schrift „nur" dazu da ist, die Sprache in ein anderes Medium zu transportieren, wurde gewaltig gerüttelt (ebd.). Wie die Einführung der Schrift und die massive Reproduktion von Texten die Kommunikation der Gesellschaft verändert hatte, zeigt Ong in seinem Buch „Mündlichkeit und Schriftlichkeit“ (1987) und ganz besonders in dem vierten Kapitel „Das Schreiben konstruiert das Denken neu“. Allerdings findet man diese Perspektive bereits bei Wygotsky (1964: 224ff.):

Die schriftliche Sprache setzt [...] einen hohen Grad der Abstraktion voraus. [...] Sie ist eine Sprache ohne Gesprächspartner [...]. Bei der schriftlichen Sprache ist der, an den die Sprache gerichtet ist, entweder überhaupt nicht da oder hat mit dem Schreibenden keinen Kontakt. Es ist eine Monolog-Sprache, das Gespräch mit weißem Blatt Papier, mit einem eingebildeten oder nur vorgestellten Gesprächspartner [...].

Heutzutage wird Schriftlichkeit in der Forschung als ein autonomes System angesehen, das wesentliche Unterschiede in den linguistischen Merkmalen im Vergleich zum mündlichen Sprachgebrauch aufweist (Fix 2008: 64).

Olson (1977) hebt hervor, dass die Struktur der Schriftsprache in Syntax, Semantik, Aufbau und Inhalt komplexer und differenzierter ist, und zwar aufgrund dessen, dass der Textzusammenhang vorwiegend mit sprachlichen Mitteln und in einer isolierten Situation hergestellt wird, und des Weiteren gelte es zu bedenken, dass schriftliche Äußerungen einen höheren Verbindlichkeitsgrad als mündliche aufweisen. Auch Wolff weist auf diese Unterschiede hin und unterstreicht, dass in jeder Sprache, die verschriftet werden kann, zwei unterschiedliche Sprachnormen vorhanden sind. Die gesprochene Sprache setze dynamisches Antworten voraus, in der geschriebenen Sprache dagegen wirke man eher statisch. Die letztere sei konservativer, während die erstere ständigen Änderungen unterliege. Der Schreiber sei gezwungen, eine Form von Diskurs zu entwickeln, die durch explizite Referenz und durch logisch aufeinander bezogene Aussagen charakterisiert ist, wogegen der Sprecher dazu weniger verpflichtet sei (Wolff 2002: 196f.).

Unter den Termini "gesprochen/mündlich" und "geschrieben/schriftlich" wird oft lediglich die Art der materiellen Realisierung der sprachlichen Äußerungen/des Textes verstanden, d.h., dass sich diese in Form von Lauten (phonisch) oder in Form von Schriftzeichen (graphisch) manifestieren (vgl. Maiworm 2003: 15). Denkt man jedoch an einige phonisch dargebotene 
Sprachrealisierungen wie Vorträge oder Reden, die zwar medial mündlich begegnen, doch durch ihre komplexen sprachlichen Strukturen eher der Konzeption schriftlicher Texte entsprechen, oder betrachtet man private Briefe, E-Mails oder Äußerungen im Chat, die im Medium der Schrift verankert, jedoch mit der Vorstellung von ,Schriftlichkeit' nur schwer vereinbar sind, dann erweist sich eine solche Unterscheidung als unzureichend (vgl. Fix 2008: 67).

Günther weist in diesem Zusammenhang darauf hin, dass eine eindimensionale Abbildung der Dichotomie Schriftlichkeit vs. Mündlichkeit auf andere Gegensatzpaare wie hypotaktisch vs. parataktisch, monologisch vs. dialogisch, reflektiert vs. spontan dem Gegenstand nicht gerecht wird (Günther 2010: 11ff.). In der sprachwissenschaftlichen Schriftlichkeitsforschung setzte vor einiger Zeit eine Neuorientierung ein, die eben auf dieser Erkenntnis beruht.

Mündlichkeit und Schriftlichkeit unterscheiden sich nämlich voneinander auch durch ihre Konzeption. Deshalb sprechen Koch und Oesterreicher (1985) in Anlehnung an Söll (1985) von einer medialen und einer konzeptionellen Dimension: Ein Text kann zwar im Medium Schrift präsentiert werden, aber von seiner Konzeption an der Mündlichkeit orientiert sein und umgekehrt. Bei der konzeptionellen Mündlichkeit und der konzeptionellen Schriftlichkeit spielen die Kommunikationsbedingungen und Versprachlichungsstrategien eine wichtige Rolle (Koch, Oesterreicher 1990: 8 ff.). Die Kommunikationsbedingungen ergeben sich unter anderem aus der Anzahl der Rezipienten, daraus, ob das Thema festgelegt ist, wie spontan der Text realisiert wird, wie hoch die Spontaneität, die Emotionalität und die Vertrautheit der Kommunikationspartner einzustufen ist. Je mehr Rezipienten es gibt, je freier das Thema, je spontaner und emotioneller die Äußerungen, je größer der Vertrautheitsgrad, desto stärker ist die Tendenz zur konzeptionellen Mündlichkeit. Das umgekehrte Verhältnis würde von einer konzeptionellen Schriftlichkeit zeugen.

Der Planungsgrad des Textes ist eine Versprachlichungsstrategie, aufgrund deren seine Zugehörigkeit zur konzeptionellen Mündlichkeit oder zur konzeptionellen Schriftlichkeit vermutet werden kann. Je weniger Zeit für die Produktion des Textes zur Verfügung steht, desto geringer ist die Informationsdichte, desto öfter kommt es zu Wiederholungen, und die Unvollständigkeit ist für solche Texte charakteristisch. Beim Vorliegen solcher Merkmale haben wir mit konzeptioneller Mündlichkeit zu tun. Gibt es ausreichend Zeit für die Produktion, für die Revisionen und Korrekturen, ist der Text im Hinblick sowohl auf den Inhalt als auch auf die verwendeten Strukturen komplexer und entspricht so den Prinzipien der konzeptionellen Schriftlichkeit (Koch, Oesterreicher 1990: 11).

Zwischen den Polen Nähe und Distanz, zwischen konzeptionell eher mündlich oder schriftlich gibt es verschiedene Ausprägungsgrade der Merkmale, was Merz-Grötsch (2000: 36f.) in Anlehnung an Koch und Oesterreicher (1994) graphisch wie folgt darstellt: 
Tabelle 1. Merz-Grötsch 2000: 36

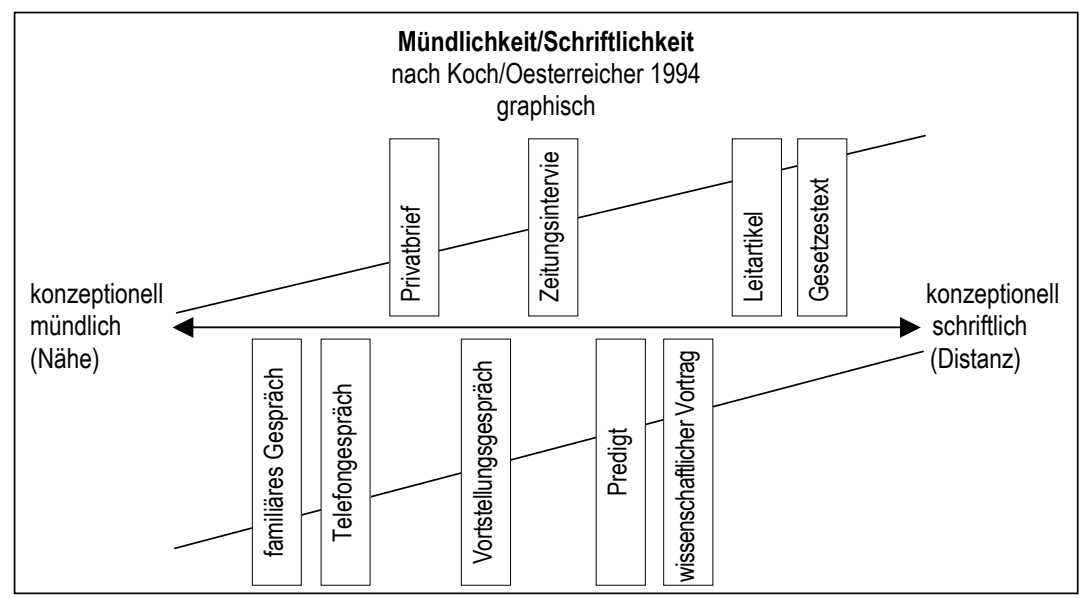

Texte können sich voneinander auf allen (text)linguistischen Ebenen unterscheiden. Fix (2008: 65ff) fasst die Unterschiede folgendermaßen tabellarisch und übersichtlich zusammen:

Tabelle 2. Fix 2008: 65

\begin{tabular}{|c|c|c|}
\hline $\begin{array}{l}\text { Linguistische } \\
\text { Ebene }\end{array}$ & Mündlichkeit (Oralität) & Schriftlichkeit (Literalität) \\
\hline 1 & 2 & 3 \\
\hline $\begin{array}{l}\text { pragmatisch } \\
\text { (Sprachhandeln) }\end{array}$ & $\begin{array}{l}\text { Anwesenheit beider Kommunikations- } \\
\text { partner in der Situation, Sprecherwechsel } \\
\text { möglich, gegenseitiges Reagieren } \\
\text { Nonverbale Kontaktsignale z.B. Mimik, } \\
\text { Gestik; paraverbale Mittel, z.B. Stimmdy- } \\
\text { namik, Betonung, Sprechmelodie, aber } \\
\text { auch Einschübe wie „mhm“, dadurch } \\
\text { Abtönungsmöglichkeiten } \\
\text { - offene Struktur } \\
\text { - Wiederholungen } \\
\text { - spontaner, assoziativer } \\
\text { - emotionaler } \\
\text { - Sozio- und Dialekte verfügbar } \\
\text { - Möglichkeit nur zu unmittelbaren } \\
\text { Reparaturen } \\
\text { - akustische Realisierung, in der Regel } \\
\text { schnell vergänglich }\end{array}$ & $\begin{array}{l}\text { Situationsentbunden (nur hypothetische } \\
\text { Interaktion zwischen Autor und Leser, } \\
\text { Produktion und Rezeption nicht si- } \\
\text { multan, Rektion nur zeitlich versetzt } \\
\text { möglich } \\
\text { Kontaktsignale fehlen (Prosodie hat in } \\
\text { Graphie keine Entsprechung, nur } \\
\text { Interpunktion als Signal vorhanden } \\
\text { - geschlossene Struktur } \\
\text { - Vermeidung von Redundanz } \\
\text { - planbarer, kognitiv reflektierter } \\
\text { - stärkere Distanzierung von Emotio- } \\
\text { nen } \\
\text { - Standardsprache } \\
\text { - Möglichkeit zur späteren Revision } \\
\text { - nachträglich revidierbar } \\
\text { - Kodierung in Schrift, konserviert }\end{array}$ \\
\hline $\begin{array}{l}\text { sprachsystematisch } \\
\text { phonetisch } \\
\text { und phonemisch }\end{array}$ & phonetische Varianten im Mündlichen & Phonem-Graphem-Diskrepanz \\
\hline
\end{tabular}


tab. 2

\begin{tabular}{|l|l|l|}
\hline \multicolumn{1}{|c|}{1} & \multicolumn{1}{|c|}{2} & \multicolumn{1}{|c|}{3} \\
\hline $\begin{array}{l}\text { grammatisch } \\
\text { (morphologisch, } \\
\text { syntaktisch) }\end{array}$ & $\begin{array}{l}\text { Endungsreduktion } \\
\text { einfache, variationsarme Syntax (6-8 } \\
\text { Wörter-Einheiten), reihende Struktur } \\
\text { (parataktische Konstruktion) } \\
\text { weniger Zeitformen (v.a. Präsens, Perfekt) } \\
\text { Ellipsen, Abbrüche, Redundanzen } \\
\text { Freie Wortstellung } \\
\text { weniger Informationsgehalt im Verhältnis } \\
\text { zur Textlänge }\end{array}$ & $\begin{array}{l}\text { hoher Explizitheitsgrad } \\
\text { komplexere, elaboriertere grammatische } \\
\text { Strukturen } \\
\text { längere Sätze, unterordnende Struktur } \\
\text { (mehr hypotaktische Konstruktionen) } \\
\text { vollständige Sätze, Vermeidung von } \\
\text { Redundanz, feste Wortstellung } \\
\text { verdichtete Information, abstrakt }\end{array}$ \\
\hline lexikalisch & einfachere Lexik & $\begin{array}{l}\text { größere Wortschatzvarianz, schwie- } \\
\text { rigere Lexik }\end{array}$ \\
\hline textuell & $\begin{array}{l}\text { offene, meist dialogische Textstruktur, die } \\
\text { auch Kohärenzbrüche zulässt }\end{array}$ & $\begin{array}{l}\text { stärkere Strukturiertheit durch Ko- } \\
\text { härenz und konsequenten Kohäsions- } \\
\text { mittelgebrauch }\end{array}$ \\
\hline
\end{tabular}

Da die Grenzen und die Übergänge zwischen Mündlichkeit und Schriftlichkeit fließend sind, weist Fix zu Recht darauf hin, dass diese Zusammenstellung nur eine Hilfskonstruktion ist. Fasst man das oben Dargestellte zusammen, dann besteht der schwerwiegendste und wesentlichste Unterschied zwischen mündlich und schriftlich produzierten Texten darin, dass beim Verfassen schriftlicher Texte ein höherer Grad an Abstraktion und an analytischen Fähigkeiten gefordert wird, und zwar deshalb, weil die Verstehensmöglichkeit trotz Abwesenheit des Partners bzw. Lesers begründet werden muss (Knoop 1983: 24). Um schriftliche Texte erstellen zu können, muss in der Regel ein zweites Register der Kommunikation ausgebildet werden, wozu man ein entsprechend entwickeltes und speziell darauf ausgerichtetes Sprachbewusstsein braucht.

An dieser Stelle wird der Versuch unternommen, aufzuzeigen, inwieweit die Funktionen der Schriftsprache und die Unterschiede zwischen der gesprochenen und geschriebenen Sprache den Lernern des Deutschen als Fremdsprache - genauer gesagt den Erstsemestern der Angewandten Linguistik an der Maria Curie-Skłodowska-Universität zu Lublin - bewusst sind. Die im Folgenden angeführten Daten sind Teil einer größeren Studie an der 40 Deutschlerner nach Abschluss der Oberschule und Aufnahme eines linguistischen Studiums teilgenommen haben und in deren Verlauf unter anderem eine etwa 40 Fragen umfassende Untersuchung durchgeführt wurde, die darauf abzielte, die Erfahrungen der Erstsemester im Bereich der fremdsprachlichen Schreibfertigkeitsentwicklung zu ermitteln. Die Untersuchung wurde von der Autorin des vorliegenden Beitrags geplant und durchgeführt. 
Bei einer der Fragen wurde nach den Unterschieden zwischen gesprochener und geschriebener Sprache/Mündlichkeit und Schriftlichkeit sowie nach den Zwecken und Funktionen des Schreibens und den Situationen, in denen die Probanden das Medium Schreiben einsetzen, geforscht.

Die häufigsten Antworten in Bezug auf die Unterschiede seien wie folgt zusammengestellt:

Tabelle 3. Eigene Bearbeitung

\begin{tabular}{|c|c|}
\hline Mündlichkeit & Schriftlichkeit \\
\hline 1 & 2 \\
\hline $\begin{array}{l}2 \text { Personen: } \\
\text { verflüchtigt sich schneller } \\
\text { verändert sich }\end{array}$ & $\begin{array}{l}5 \text { Personen: } \\
\text { - verflüchtigt sich nicht so schnell, bleibt } \\
\text { - wenn man schreibt, sind alle Unzulänglich- } \\
\text { keiten sichtbar bis auf die Aussprache }\end{array}$ \\
\hline $\begin{array}{l}19 \text { Personen: } \\
\text { - verlangt keine perfekte Grammatik oder } \\
\text { Wortschatz/Lexik } \\
\text { - entspricht nicht immer den grammati- } \\
\text { schen Regeln, } \\
\text { - ist weniger korrekt in puncto Grammatik, } \\
\text { enthält mehr Fehler, ist oft nicht genau, man } \\
\text { verstößt öfter gegen grammatische Regeln } \\
\text { - man konzentriert sich beim Sprechen } \\
\text { weniger auf Grammatik, grammatische } \\
\text { Unzulänglichkeiten werden eher toleriert, } \\
\text { man hat keine Zeit für Fehlerkorrektur } \\
\text { - das Hauptziel ist die Vermittlung der } \\
\text { Information } \\
\text { - am wichtigsten ist die Flüssigkeit und dass } \\
\text { der Sprecher verstanden wird }\end{array}$ & $\begin{array}{l}12 \text { Personen: } \\
\text { - typisch für Wissenschaftssprache } \\
\text { - formell } \\
\text { - keine Umgangssprache } \\
\end{array}$ \\
\hline $\begin{array}{l}16 \text { Personen: } \\
\text { - höherer Grad an Spontaneität } \\
\text { - ist lockerer } \\
\text { - man hat weniger Zeit für das Finden } \\
\text { richtiger Ausdrücke und wenig Zeit für } \\
\text { die Bildung der Aussage }\end{array}$ & $\begin{array}{l}\text { 11 Personen: } \\
\text { - geschrieben wird nach bestimmten Regeln, } \\
\text { Struktur und Komposition ist wichtig } \\
\text { - man muss sich an bestimmte Regeln beim } \\
\text { Schreiben halten } \\
\text { - logisch, sorgfältig } \\
\mathbf{1 7} \text { Personen: } \\
\text { - man kann länger überlegen und Fehler kön- } \\
\text { nen korrigiert werden } \\
\text { - Zeit zum Nachdenken, deshalb weniger Fehler, } \\
\text { aber höhere Komplexität des Wortschatzes } \\
\text { und der Strukturen } \\
\text { - überlegt, geplant } \\
\text { - man kann genauer sein, weil man mehr Zeit } \\
\text { hat, um sich Gedanken zu machen } \\
\text { - Hauptziel - die Vermittlung der Information } \\
\text { auf eine korrekte Art und Weise }\end{array}$ \\
\hline
\end{tabular}


tab. 3

\begin{tabular}{|c|c|}
\hline 1 & 2 \\
\hline $\begin{array}{l}20 \text { Personen: } \\
\text { - einfache Formulierungen } \\
\text { - einfacherer Wortschatz und kürzere Sätze, } \\
\text { weniger komplex, einfachere Komposition, } \\
\text { Sätze sind weniger präzise und manchmal } \\
\text { lässig } \\
\text { - Gedankensprünge und Abkürzungen } \\
\text { - etwas chaotisch } \\
\text { - häufigere Wiederholungen }\end{array}$ & $\begin{array}{l}12 \text { Personen: } \\
\text { Komplexität } \\
\text { - reicher, umfangreicher Wortschatz } \\
\text { - komplexere Wendungen, pathetischer Wort- } \\
\text { schatz, muss stilistisch besser aufgebaut sein } \\
\text { - komplexe Sätze und Strukturen, gramma- } \\
\text { tisch sehr fortgeschrittene Formulierungen } \\
\text { - weniger Wiederholungen }\end{array}$ \\
\hline $\begin{array}{l}10 \text { Personen: } \\
\text { - umgangssprachlich, Slang, Dialekt }\end{array}$ & $\begin{array}{l}2 \text { Personen: } \\
\text { - ohne Kontext, der Kontext muss geschildert } \\
\text { werden, denn die Situation kann ihn nicht } \\
\text { bestimmen }\end{array}$ \\
\hline
\end{tabular}

Aus dieser Zusammenstellung kann die Folgerung abgeleitet werden, dass den Lernern die grundlegenden Unterschiede zwischen Mündlichkeit und Schriftlichkeit durchaus bewusst sind:

- 21 Personen $=52,5 \%$ unterstreichen, dass für die gesprochene Sprache einfachere Formulierungen und Wiederholungen charakteristisch sind, dass man sich weniger präzise ausdrückt, weil durch die Situation vieles deutlich wird;

- 18 Personen $=48 \%$ weisen z.B. auf die Tatsache hin, dass man im Falle von geschriebener Sprache mehr Zeit zur Verfügung hat, womit die Möglichkeit geboten wird, den Text zu korrigieren;

- 16 Personen $=40 \%$ nennen den höheren Grad an Spontaneität der gesprochenen im Vergleich zur geschriebenen Sprache;

- 11 Personen $=27,5 \%$ weisen auf die Notwendigkeit des genaueren, regelgemäßen Ausdrucks hin;

- 12 Personen $=30 \%$ nennen einen höheren Grad an Komplexität bei der geschriebenen Sprache.

Doch kann dies nicht darüber hinwegtäuschen, dass einige wichtige Unterschiede wie z.B. die Situationsentbundenheit, das Fehlen von Kontaktsignalen oder die stärkere Distanzierung von Emotionen im Falle des geschriebenen Wortes nur von vereinzelten Personen erkannt werden und es an konkreten Beispielen in Bezug etwa auf die oft genannten Eigenschaften wie komplexere Strukturen oder kompliziertere Grammatik mangelt. Aufgrund der Nennungen der Befragten kann man den Eindruck gewinnen, dass die schriftliche Sprache im Vergleich zur mündlichen von ihnen als die schwierigere, anspruchsvollere und höhere Anforderungen an den Schreibenden 
stellende Variante angesehen wird, die jedoch dadurch, dass man beim Schreiben mehr Zeit hat, weniger Angst hervorruft.

Bereits nach Abschluss der vierten Bildungsetappe sollten die Schüler, insbesondere die, die das erweiterte Abitur ablegen wollen - der alten wie der neuen Programmgrundlage für die Fremdsprachen zufolge - in der Lage sein, entsprechende Stile beim Sprechen wie auch beim Schreiben einzusetzen und zwischen formeller und informeller Sprache $\mathrm{zu}$ differenzieren. Demzufolge hätte man erwartet, dass in diesem Zusammenhang im Unterricht auf die Unterschiede zwischen der gesprochenen und geschriebenen Sprache ebenfalls eingegangen wird. Den Antworten der Probanden ist jedoch zu entnehmen, dass im schulischen DaF-Unterricht die Unterschiede zwischen gesprochener und geschriebener Sprache nicht thematisiert werden - eine solche Antwort gaben 28 Probanden. Etwas besser sieht es im Polnischunterricht aus: Hierzu meinten 24 Probanden, dass der Lehrer auf die Unterschiede hingewiesen und sie besprochen hat, wodurch die Schüler im DaF-Unterricht auf die Kenntnisse aus der Muttersprache zurückgreifen konnten. Meines Erachtens sollte der Frage, warum im Rahmen des Deutschunterrichts auf diese Unterschiede nicht eingegangen wird, verstärkt nachgegangen werden. Ich beabsichtige mit diesem Postulat nicht dafür zu plädieren, dass man an den Schulen im Fremdsprachenunterricht ein regelrechtes textlinguistisches Seminar bzw. Vorträge vorsieht, dennoch stehe ich auf dem Standpunkt, dass das Wissen der Probanden in diesem Bereich unter besonderer Berücksichtigung der kulturellen Besonderheiten im Fremdsprachenunterricht explizit thematisiert und nicht bloß angedeutet werden sollte. Für Studierende einer philologischen Fachrichtung, wo es sowohl auf die rezeptive als auch die produktive Auseinandersetzung mit mündlichen und schriftlichen Texten besonders ankommt, ist auch das Wissen um die zwischen ihnen auftretenden Unterschiede unabdingbar. Als zukünftige Deutschlehrer und/oder Übersetzer benötigt gerade diese Zielgruppe ein fundiertes Wissen in diesem Bereich, was in den Kompositionsübungen im Rahmen des linguistischen Studiums unbedingt berücksichtigt werden sollte, indem man dort den Hebel ansetzt und den Studierenden geeignete Möglichkeiten und Wege, die Wissenslücken zu füllen, aufzeigt.

Schreiben bedeutet nicht einfach Gesprochenes in ein anderes Medium umzusetzen. Diese Feststellung klingt wie eine Binsenweisheit, dennoch kann man sich des Eindrucks nicht erwehren, dass nicht alle Studierenden sie ernst nehmen. Von daher ist es dringend notwendig, auf die Unterschiede zwischen mündlicher und schriftlicher Kommunikation noch stärker aufmerksam zu machen, auch schon auf den niedrigeren Stufen. Im Schreibunterricht mit den Linguistik- oder Germanistikstudierenden ist dies besonders wichtig, zumal es sich in ihrem Fall um einen Adressatenkreis handelt, 
der in Zukunft als Lehrer oder Übersetzer/Dolmetscher tätig sein will. Und in diesen Berufen sind sie ohne Zweifel auf das Wissen, das es ihnen erlaubt, die richtigen Entscheidungen bezüglich Abfassung der für die jeweilige Situation relevanten Texte zu treffen, angewiesen. Allerdings sollte man sich in der Didaktik des Schreibens - meines Erachtens insbesondere eben im Rahmen eines linguistischen Studiums - nicht nur auf die mediale Ebene konzentrieren, die auch noch heute oft die Unterrichtspraxis beherrscht. Im Schreibunterricht sollten die Lerner das Schreiben lernen, das heißt, sie sollen in die Lage versetzt und dazu befähigt werden - und hier stimme ich Günther (2010: 17) völlig zu - „sich in konzeptioneller Schriftlichkeit zurechtzufinden - also etwa situationsentbunden zu formulieren, die Reaktion des Lesers zu antizipieren, die Möglichkeit des Redigierens immer schon beim Schreiben zu bedenken." Die schulische Praxis versagt in diesem Bereich besonders kläglich, denn „das Verbessern, Durchstreichen, Neuansetzen hat noch immer das Odium des Unvollkommenen, des teilweisen Versagens" (ebd.). Günther bezieht sich in seinen diesbezüglichen Ausführungen zwar auf den muttersprachlichen Unterricht, doch gelten sie meiner Ansicht nach erst recht für den Fremdsprachenunterricht. Dabei sollte das Überprüfen und Redigieren eines schriftlichen Textes mehr Beachtung finden, da dies bedeutende Kennzeichen entwickelter Schreibfertigkeit sind, was auch gängige Modelle des Schreibprozesses (Fix 2008; Merz-Grötsch 2000) klarlegen.

\section{LITERATURVERZEICHNIS}

Bloomfield, L., 1933. Language. London: Motilal Banarsidass.

Bußmann, H. (Hrsg.), 1983. Lexikon der Sprachwissenschaft. 1. Aufl. Stuttgart: Kröner.

Fix, M., 2008. Texte schreiben. Schreibprozesse im Deutschunterricht. Stuttgart: UTB Verlag.

Günther, H., 2010. Beiträge zur Didaktik der Schriftlichkeit (= Kölner Beiträge zur Sprachdidaktik 6). Duisburg: Gilles \& Francke Verlag.

Knoop, U., 1983. Mündlichkeit und Schriftlichkeit. Vorläufige Überlegungen zu ihrem Status. In: Grosse, S. (Hrsg.) Schriftsprachlichkeit. Düsseldorf: Schwann, 24-36.

Koch, P., 1992. Schriftlichkeit im Fremdsprachenunterricht. In: Börner, W., Vogel, K. (Hrsg.). Schreiben in der Fremdsprache. Prozess und Text, Lehren und Lernen. Bochum: AKS, 2-28.

Koch, P., Oesterreicher, W., 1985. Sprache der Nähe - Sprache der Distanz - Mündlichkeit und Schriftlichkeit im Spannungsfeld von Sprachtheorie und Sprachgeschichte. In: Romanistisches Jahrbuch, 36, 15-43.

Koch, P., Oesterreicher, W., 1990. Gesprochene Sprache in der Romania: Französisch, Italienisch, Spanisch. Tübingen: Niemeyer.

Koch, P., Oesterreicher, W., 1994. Schriftlichkeit und Sprache. In: Günther, H., Ludwig, O. (Hrsg.). Schrift und Schriftlichkeit/Writing and its Use. Ein interdisziplinäres Handbuch internationaler Forschung/An Interdisciplinary Handbook of International Research. Band 1. Berlin, New York: de Gruyter, 587-604. 
Maiworm, S., 2003. Zwischen Mündlichkeit und Schriftlichkeit: Eine linguistische Analyse französischsprachiger Chats. http://docserv.uni-duesseldorf.de/servlets/DerivateServlet/Derivate2808/808.pdf (abgerufen am: 14.10.2012).

Merz-Grötsch, J., 2000. Schreiben als System. Band 1: Schreibforschung und Schreibdidaktik: ein Überblick. Freiburg i.Br.: Fillibach Verlag.

Merz-Grötsch, J., 2001. Schreiben als System. Band 2: Die Wirklichkeit aus Schülersicht: eine empirische Analyse. Freiburg i.Br.: Fillibach Verlag.

Olson, D., 1977. From utterance to text. In: Harvard Educational Review, 47, 257-279.

Ong, W.S., 1987. Oralität und Literalität - Die Technologisierung des Wortes. Opladen: Westdeutscher Verlag.

Söll, L., 1985. Gesprochenes und geschriebenes Französisch. Berlin: Schmidt.

Wolff, D., 2002. Fremdsprachenlernen als Konstruktion. Grundlagen für eine konstruktivistische Fremdsprachendidaktik. Frankfurt am Main. u.a.: Lang.

Wygotski, L.S., 1964. Denken und Sprechen. Berlin: Fischer. 